\title{
Acknowledgement of Reviewers 2016
}

Published online: 14 January 2017

(C) Springer Science+Business Media New York 2017

The Editor, Eric Rowinsky, and the Associate Editors would like to acknowledge the valuable contributions of the reviewers to the journal. We fully understand the burden and effort that are involved in the reviews and are very grateful for your support. We rely on your expertise to maintain the standards of excellent science for the journal, Investigational New Drugs.

\author{
Yasushi Adachi \\ Barbara Altieri \\ Kyaw Aung \\ Koichi Azuma \\ Jan Beumer \\ Giampaolo Bianchini \\ Javier Blanco \\ P. Callery \\ E. Calvino \\ Ralph Chebib \\ Vincent Chung \\ Kristen Ciombor \\ Wilson Clements \\ Romain Coriat \\ Denise de Oliveira Silva \\ Roberto Dominguez \\ R. Donald Harvey \\ Afshin Dowlati \\ Hélène Elleaume \\ Matthew J. Ellis \\ Bratko Filipič \\ Mashide Fukudo \\ Ariela Fundia \\ Jessica Geiger \\ Marwan Ghosn \\ Beppe Giaccone \\ Francis Giles
}

Lia Gore
James Groome
Eiki Ichihara
Koji Izutsu
Angeles Juarranz
Masahi Kanai
Katie Kelley
Christian Klein
Vincent Launay-Vacher
Christophe LeTourneau
Chia-Chi (Josh) Lin
M. Lolkema
Harry Lybeck
Flamini Marina Ines
Hironobu Minami
Olivier Mir
Manoj Mishra
Monica Mita
Susan Mooberry
Aung Naing
David Naor
Andrea Necchi
Naoyuki Nogami
Mukesh Nyati
Ingo Ott
Neven Patrick
Philip Philip

Wenbin Qian

Tadeusz Robak

Marta Romero

Charles J. Rosser

Benoit Rousseau

Yuval Shaked

Guru Sonpavde

Toru Sugiyama

Young Suk Park

Matthew Sydes

Stefan Symeonides

Andreas Tzakos

Brian Van Tine

Nevena Veljkovic

Singurd Vitols

Dan von Hoff

J. Watanabe

Glen Weiss

Robert Wesolowski

Christian Woloch

Nigel Wynn

K. Yamada

Gokhan Zengin

Xiaofeng Zhu 